\title{
Entropy and the hyperplane conjecture in convex geometry
}

\author{
Sergey Bobkov \\ School of Mathematics \\ University of Minnesota \\ 206 Church St. S.E. \\ Minneapolis, MN 55455 USA. \\ Email: bobkov@math.umn.edu
}

\author{
Mokshay Madiman \\ Department of Statistics \\ Yale University \\ 24 Hillhouse Avenue \\ New Haven, CT 06511, USA. \\ Email: mokshay.madiman@yale.edu
}

\begin{abstract}
The hyperplane conjecture is a major unsolved problem in high-dimensional convex geometry that has attracted much attention in the geometric and functional analysis literature. It asserts that there exists a universal constant $c$ such that for any convex set $K$ of unit volume in any dimension, there exists a hyperplane $H$ passing through its centroid such that the volume of the section $K \cap H$ is bounded below by $c$. A new formulation of this conjecture is given in purely information-theoretic terms. Specifically, the hyperplane conjecture is shown to be equivalent to the assertion that all log-concave probability measures are at most a bounded distance away from Gaussianity, where distance is measured by relative entropy per coordinate. It is also shown that the entropy per coordinate in a log-concave random vector of any dimension with given density at the mode has a range of just 1. Applications, such as a novel reverse entropy power inequality, are mentioned.
\end{abstract}

\section{INTRODUCTION}

The hyperplane conjecture (sometimes called the slicing problem) was originally raised by J. Bourgain [1] in 1986 and has attracted a lot of attention since. In one basic formulation, this may be stated as follows.

Conjecture 1: [SLICING Form OF HyPERPLANE CONJECTURE] There exists a universal, positive constant $c$ (not depending on $n$ ) such that for any convex set $K$ of unit volume in $\mathbb{R}^{n}$, there exists a hyperplane $H$ such that the $(n-1)$ dimensional volume of the section $K \cap H$ is bounded below by $c$.

There are several equivalent formulations of the conjecture, all of a geometric or functional analytic flavor. For instance, another popular formulation (developed by K. Ball [2]) is that the isotropic constant (to be defined later) of a logconcave measure in any Euclidean space is bounded above by a universal constant independent of dimension.

The main goal of this note is to demonstrate that the hyperplane conjecture has a formulation in purely informationtheoretic terms. First we need to recall the notion of a logconcave density. A probability density function (or simply "density") $f$ defined on the linear space $\mathbf{R}^{n}$ is said to be log-concave if

$$
f(\alpha x+(1-\alpha) y) \geq f(x)^{\alpha} f(y)^{1-\alpha}
$$

for each $x, y \in \mathbb{R}^{n}$ and each $0 \leq \alpha \leq 1$. If $f$ is log-concave, we will also use the adjective "log-concave" for a random variable $X$ distributed according to $f$, and for the probability measure induced by it.

Log concavity has been deeply studied in probability, statistics, optimization and geometry, and there are a number of results that show that log-concave random vectors resemble Gaussian random vectors. For instance, several functional inequalities that hold for Gaussians also hold for appropriate subclasses of log-concave distributions. Observe that this is not at all obvious at first glance- log-concave probability measures include a large variety of distributions including the uniform distribution on any compact, convex set, the (onesided) exponential distribution, and of course any Gaussian. Both the results and conjectures in this note are, in some sense, attempts to give a strong (quantitative) information-theoretic basis to the intuition that log-concave distributions resemble Gaussian distributions.

For a random vector $X=\left(X_{1}, \ldots, X_{n}\right)$ in $\mathbf{R}^{n}$ with density $f(x)$, let $h(X)$ or $h(f)$ denote its differential entropy, and let $D(X)$ or $D(f)$ denote its relative entropy from Gaussianity, i.e.,

$$
D(X)=h(Z)-h(X),
$$

where $Z$ has the same covariance matrix as $X$. For instance, for dimension $n=1$,

$$
D(X)=\frac{1}{2} \log [2 \pi e \operatorname{Var}(X)]-h(X) .
$$

Conjecture 2: [ENTROPIC FORM OF HYPERPLANE CONJECTURE] For any log-concave density $f$ on $\mathbb{R}^{n}$ and some universal constant $c$,

$$
\frac{D(f)}{n} \leq c
$$

This formulation has several advantages. First, it demonstrates deep and hitherto not well explored links between information theory and convex geometry. Second, it allows for the potential deployment of information-theoretic tools for attacking open problems such as this conjecture in convex geometry. Third, the information-theoretic formulation is very 
natural, and along the way to obtaining it, we also provide new bounds on the differential entropy of log-concave probability measures that are of intrinsic interest, and have a number of applications in their own right.

In particular, Conjecture 2 gives a pleasing formulation of the slicing problem as a statement about the (dimension-free) closeness of an arbitrary log-concave measure to Gaussian measure. It is possible to prove, using results from [3], that $D(X)$ is bounded above by a constant for one-dimensional log-concave distributions (see [4]). Thus Conjecture 2 posits that an arbitrary multivariate log-concave distribution cannot get very much more non-Gaussian than a product of onedimensional log-concave distributions.

The proof of the equivalence of Conjectures 1 and 2 relies on a remarkable entropy comparison inequality. Specifically, in terms of the "amount of randomness" as measured by entropy per coordinate, any log-concave random vector of any dimension contains randomness that differs from that in the normal random variable with the same maximal density value by at most $1 / 2$.

Theorem 1: If a random vector $X$ in $\mathbf{R}^{n}$ has a log-concave density $f$, let $Z$ in $\mathbb{R}^{n}$ be any normally distributed random vector with maximum density being the same as that of $X$. Then

$$
\frac{1}{n} h(Z)-\frac{1}{2} \leq \frac{1}{n} h(X) \leq \frac{1}{n} h(Z)+\frac{1}{2} .
$$

Equality holds in the lower bound if and only if $X$ is uniformly distributed on a convex set with non-empty interior. Equality holds in the upper bound if $X$ has coordinates that are i.i.d. exponentially distributed.

Theorem 1 and Conjecture 2 have an intriguing relationship. Indeed, the latter says that for any log-concave random vector $X$ taking values in $\mathbb{R}^{n}$,

$$
\frac{1}{n} h(X) \geq \frac{1}{n} h(Z)-C,
$$

where $C$ is a universal constant, and $Z$ is the normal with the same covariance matrix as $X$. The inequality (2) looks like the lower bound of Theorem 1, except that the way in which the matching Gaussian is chosen is to match the covariance matrix rather than the maximum density. Thus, while Theorem 1 is a Gaussian comparison result for log-concave random vectors where the Gaussian is chosen by matching the supremum norm, our formulation of the hyperplane conjecture is a Gaussian comparison result for log-concave random vectors where the Gaussian is chosen by matching the mean and covariance matrix.

This note is organized as follows. In Section II, we prove entropy bounds for log-concave probability measures, and in particular, Theorem 1. In Section III, we prove the equivalence of Conjectures 1 and 2. In Section IV, we summarize some possible extensions of the results in this note. We end in Section V with some discussion of applications of our results.

\section{ENTROPY BOUNDS FOR LOG-CONCAVE MEASURES}

This result follows quite easily from the following basic proposition. Throughout this paper, we will write $\|f\|=$

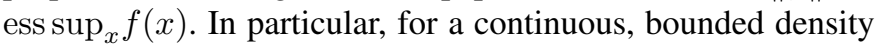
$f$, this is just the maximum value of $f$.

Proposition 1: If a random vector $X$ in $\mathbf{R}^{n}$ has density $f$, then

$$
\frac{1}{n} h(X) \geq \log \|f\|^{-1 / n} .
$$

If, in addition, $f$ is log-concave, then

$$
\frac{1}{n} h(X) \leq 1+\log \|f\|^{-1 / n},
$$

with equality for the $n$-dimensional exponential distribution, concentrated on the positive orthant with density $f(x)=$ $e^{-\left(x_{1}+\cdots+x_{n}\right)}, x_{i}>0$.

Proof: The lower bound is trivial and holds without any assumption on the density:

$$
h(X) \geq \int_{\mathbf{R}^{n}} f(x) \log \frac{1}{\|f\|} d x=\log \frac{1}{\|f\|} .
$$

Let us derive the upper bound. By definition of $\log$ concavity, for any $x, y \in \mathbf{R}^{n}$,

$$
f(t x+s y) \geq f(x)^{t} f(y)^{s}, t, s>0, t+s=1 .
$$

Integrating with respect to $x$, we get

$$
t^{-n} \int f(x) d x \geq f(y)^{s} \int f(x)^{t} d x .
$$

Now using the assumption that $\int f=1$ and maximizing over $y$, we obtain

$$
t^{-n} \geq\|f\|^{1-t} \int f(x)^{t} d x .
$$

Observe that the left and right sides of (3) are equal for $t=1$, and the above tells us the left side dominates the right side for $0<t \leq 1$. This implies that we can compare derivatives of the two sides of (3) in $t$ at $t=1$. Specifically, we have

$$
-n \leq-\log \|f\|+\int f(x) \log f(x) d x,
$$

which yields the desired inequality. It is easy to check that a product of exponentials is an instance of equality.

We can now complete the proof of Theorem 1.

Proof of Theorem 1. Observe that the maximum density of the $N\left(0, \sigma^{2} I\right)$ distribution is $\left(2 \pi \sigma^{2}\right)^{-n / 2}$. Thus matching the maximum density of $f$ and the isotropic normal $Z$ leads to $\left(2 \pi \sigma^{2}\right)^{1 / 2}=\|f\|^{-1 / n}$, and

$$
\frac{1}{n} h(Z)=\frac{1}{2} \log \left(2 \pi e \sigma^{2}\right)=\frac{1}{2}+\log \|f\|^{-1 / n} .
$$

Combining with Proposition 1, we are done. 


\section{An Entropic Formulation of the Slicing PROBLEM}

The main observation of this section is a relationship between the entropy distance to Gaussianity $D(f)$ and the isotropic constant $L_{f}$ for densities of convex measures.

For any probability density function $f$ on $\mathbb{R}^{n}$, let $D(f)$ be the relative entropy between $f$ and the normal density with the same mean and covariance matrix as $f$. Recall that $D(f)$ may also be written as the difference in entropies of $f$ and the normal density (see, e.g., Cover and Thomas [5]), which yields the definition given in the Introduction.

For any probability density function $f$ on $\mathbb{R}^{n}$, define its isotropic constant $L_{f}$ by

$$
L_{f}^{2}=\|f\|^{2 / n} \operatorname{det}^{\frac{1}{n}}(R) .
$$

The isotropic constant has a nice interpretation for uniform distributions on convex sets $K$. If one rescales $K$ (by a linear transformation) so that the volume of the convex set is 1 and the covariance matrix is a multiple of the identity, then $L_{K}^{2}:=$ $L_{f}^{2}$ is the value of the multiple. Such convex bodies, with volume 1 and covariance matrix a multiple of the identity, are said to be isotropic.

Observe that both $D(f)$ and $L_{f}$ are affine invariants.

Theorem 2: For any density $f$ on $\mathbb{R}^{n}$,

$$
\frac{1}{n} D(f) \leq \log \left[\sqrt{2 \pi e} L_{f}\right],
$$

with equality if and only if $f$ is the uniform density on some set of positive, finite Lebesgue measure. If $f$ is a log-concave density on $\mathbb{R}^{n}$, then

$$
\log \left[\sqrt{\frac{2 \pi}{e}} L_{f}\right] \leq \frac{1}{n} D(f),
$$

with equality if $f$ is a product of one-dimensional exponential densities.

Proof: Let $X \sim f$ have covariance matrix $R$. If $Z \sim$ $N(0, R)$,

$$
h(Z)=\frac{1}{2} \log \left[(2 \pi e)^{n} \operatorname{det}(R)\right]=\frac{n}{2} \log (C \sigma),
$$

where $\sigma=\operatorname{det}(R)^{\frac{1}{n}}$ and $C=2 \pi e$. Thus

$$
\begin{aligned}
\frac{1}{n} D(X) & =\frac{h(Z)-h(X)}{n} \\
& \leq \frac{1}{2} \log (C \sigma)-\log \|f\|^{-\frac{1}{n}} \\
& =\frac{1}{2} \log \left[C \sigma\|f\|^{2 / n}\right]=\frac{1}{2} \log \left[C L_{f}^{2}\right],
\end{aligned}
$$

and

$$
\begin{aligned}
\frac{1}{n} D(X) & =\frac{h(Z)-h(X)}{n} \\
& \geq \frac{1}{2} \log (C \sigma)-\log \|f\|^{-\frac{1}{n}}-1 \\
& =\frac{1}{2} \log \left[\frac{C}{e^{2}} \sigma\|f\|^{2 / n}\right]=\frac{1}{2} \log \left[\frac{2 \pi}{e} L_{f}^{2}\right],
\end{aligned}
$$

where the inequalities come from Proposition 1.
Theorem 2 immediately yields the following corollary, by using the fact that $D(f) \geq 0$.

Corollary 1: For any log-concave density $f$ (on Euclidean space of any dimension),

$$
L_{f} \geq \frac{1}{\sqrt{2 \pi e}} .
$$

On the other hand, whether or not this quantity, the isotropic constant $L_{f}=\|f\|^{1 / n}(\operatorname{det} R)^{1 / n}$, is bounded from above by a universal constant for the class of uniform distributions on convex bodies is precisely the hyperplane conjecture originally raised by J. Bourgain [1].

First, let us mention the following equivalences that arise from Theorem 2.

Corollary 2: Let $c(n)$ be any non-decreasing sequence, and $c^{\prime}(n)=c(n)+\frac{1}{2} \log (2 \pi e)$. Then the following statements are equivalent:

1) For any log-concave density $f$ on $\mathbb{R}^{n}, L_{f} \leq e^{c(n)}$.

2) For any log-concave density $f$ on $\mathbb{R}^{n}, D(f) \leq n c^{\prime}(n)$.

3) $\sup _{f} \min _{g} D(f \| g) \leq n c^{\prime}(n)$, where the minimum is taken over all Gaussian densities on $\mathbb{R}^{n}$, and the maximum is taken over all log-concave densities on $\mathbb{R}^{n}$.

Here the equivalence of (i) and (ii) follows from Theorem 2, and that of (ii) and (iii) follows from the easily verified fact that $D(f)=\min _{g} D(f \| g)$, where $g$ is allowed to run over all Gaussian distributions. Furthermore, the seminal paper of Hensley [6] (cf. Milman and Pajor [7]) showed that for an isotropic convex body $K$, and any hyperplane $H$ passing through its barycenter,

$$
c_{1} \leq L_{K} \operatorname{Vol}_{n-1}(K \cap H) \leq c_{2},
$$

where $c_{2}>c_{1}>0$ are universal constants. Hence the statements of Corollary 2, when restricted to uniform distributions on convex sets, are also equivalent to the statement that

$$
\operatorname{Vol}_{n-1}(K \cap H) \geq e^{-c(n)} .
$$

Thus the slicing problem or the hyperplane conjecture is simply the conjecture that $c(n)$ can be taken to be constant (independent of $n$ ), in any of the statements of Corollary 2.

Existing partial results on the slicing problem already give insight into the closeness of log-concave measures to Gaussian measures. For many years, the best known bound in the slicing problem for general bounded convex sets, due to Bourgain [8] in the centrally-symmetric case and generalized by Paouris [9] to the non-symmetric case, was

$$
L_{K} \leq c n^{1 / 4} \log (n+1) .
$$

Recently Klartag [10] removed the $\log n$ factor and showed that $L_{K} \leq c n^{1 / 4}$. Using results of Ball [2], the same bound is seen to also apply to $D(f)$, for a general log-concave density $f$. Thus we have: 
Corollary 3: There is a universal constant $c$ such that for any $\log$-concave density $f$ on $\mathbb{R}^{n}$,

$$
D(f) \leq \frac{1}{4} n \log n+c n .
$$

\section{EXTENSIONS}

While we focused on the Shannon differential entropy in this note, several of our results have generalized statements involving Rényi entropy. Proposition 1 and Theorem 1 emerge as consequences of a more general result (and rather powerful) that bounds the Rényi entropy of any order $p \geq 1$ using the maximum of the density. As a corollary of this, we show in [4] that any two Rényi entropies (or $L^{p}$-norms for different $p$ ) become comparable for the class of log-concave densities.

Another, and perhaps more interesting, extension of our results involves broadening the class of densities considered. While Proposition 1 is already remarkable in its own right, log-concavity is a relatively strong assumption, and it would be advantageous to loosen it. Inspired by this objective, one wishes to study more general classes of probability distributions, satisfying weaker convexity conditions (in comparison with log-concavity). As a natural generalization, one may consider so-called convex or hyperbolic probability measures on Euclidean spaces. In [4], we give new constraints on entropy per coordinate for this class of measures, which generalize our results under the log-concavity assumption, and expose the extremal role of multivariate Pareto-type distributions. A major advantage of considering the class of convex measures is that they can have much heavier tails than log-concave measures.

Convex measures include probability densities of the form

$$
f(x)=\varphi(x)^{-\beta}, \quad x \in \Omega,
$$

where $\varphi$ is a positive convex function on an open convex set $\Omega$ in $\mathbf{R}^{n}$, and $\beta \geq n$. The $n$-dimensional Pareto distribution with density

$$
f_{\beta}(x)=\frac{1}{Z(\beta)}\left(x_{0}+x_{1}+\cdots+x_{n}\right)^{-\beta}, \quad x_{i}>0,
$$

where $x_{0}>0$ is fixed, and where $Z(\beta)$ is a normalizing factor, belongs to this class whenever its parameter $\beta>n$.

Just for illustration, we state a generalization of Proposition 1 .

Corollary 4: For the range $\beta \geq \beta_{0} n$ with fixed $\beta_{0}>1$ (and still for $\beta \geq n+1$ ), we have

$$
\frac{1}{n} h(X) \leq C_{\beta_{0}}+\log \|f\|^{-1 / n}
$$

where the constant $C_{\beta_{0}}$ depends on $\beta_{0}$ only. However, in the larger range $\beta \geq \beta_{0}+n$ with fixed $\beta_{0} \geq 1$,

$$
\frac{1}{n} h(X) \leq \log \|f\|^{-1 / n}+O(\log n),
$$

where the $O(\log n)$ term may be explicitly bounded.

Proposition 1 is recovered in the limit as $\beta \rightarrow+\infty$.

\section{Discussion}

The results in this note have several applications. Let us call a discrete-time stochastic process $\mathbf{X}=\left(X_{i}\right)$ log-concave if all its finite-dimensional marginals are log-concave distributions. An important sub-class of the log-concave processes is the family of Gaussian processes, and this is so far the only class of processes for which the computation of entropy rate is tractable. Our inequalities give a way of obtaining some information about the entropy rate of a stationary log-concave process. One interesting class of processes where this result may be of utility, and where the study of entropy rate has attracted much recent interest, is the class of hidden Markov processes.

Getting estimates of the entropy of infinitely divisible distributions is a notoriously hard problem because of the lack of an explicit form for their density functions in most cases. It is also interesting because infinitely divisible distributions include the limiting distributions that appear in all probabilistic limit laws for sums of random variables. Our inequalities give a way of getting bounds on entropies of these distributions based on some qualitative knowledge of the shapes of their densities.

There are also other applications, such as to giving a quantitative version of an inequality of Junge concerning the behavior of $\|f\|$ on convolution. However, perhaps the most interesting appearance of these ideas is in the proof of a remarkable reverse entropy power inequality, which we mention next. First we recall some facts about the reverse Brunn-Minkowski inequality.

The reverse Brunn-Minkowski inequality is a deep result in Convex Geometry discovered by V. D. Milman in the mid 1980s (cf. [11], [12], [13], [14]). It states that, given two convex bodies $A$ and $B$ in $\mathbf{R}^{n}$, one can find linear volume preserving maps $u_{i}: \mathbf{R}^{n} \rightarrow \mathbf{R}^{n}(i=1,2)$ such that with some absolute constant $C$

$$
|\widetilde{A}+\widetilde{B}|^{1 / n} \leq C\left(|A|^{1 / n}+|B|^{1 / n}\right)
$$

where $\widetilde{A}=u_{1}(A), \widetilde{B}=u_{2}(B), \widetilde{A}+\widetilde{B}=\{x+y: x \in$ $\widetilde{A}, y \in \widetilde{B}\}$ is the Minkowski sum, and where $|A|$ stands for the $n$-dimensional volume. (Of course, one of these maps may be taken to be the identity operator.)

Note that the reverse inequality to (6),

$$
|\widetilde{A}+\widetilde{B}|^{1 / n} \geq|A|^{1 / n}+|B|^{1 / n},
$$

holds true for any such $u_{i}$ by the usual Brunn-Minkowski inequality.

Milman's inverse Brunn-Minkowski inequality has deep connections with high dimensional phenomena in convex geometry. For instance, it is known that proving Milman's inequality for convex bodies in isotropic position is equivalent to the hyperplane conjecture [15]. It has also found a number of interesting extensions and applications (cf. [16], [17], [18]).

In [19], we develop an entropic generalization of (6) that involves arbitrary log-concave probability distributions rather than just uniform measures on compact convex sets. This 
entropic generalization may be stated as an inverse of the entropy power inequality, in the same sense that Milman's inequality is an inverse of the Brunn-Minkowski inequality. Given a random vector $X$ in $\mathbf{R}^{n}$ with density $f(x)$, recall that the entropy power is defined by

$$
\mathcal{N}(X)=e^{2 h(X) / n} \text {. }
$$

In particular, if $X$ is uniformly distributed in a convex body $A \subset \mathbf{R}^{n}$, we have

$$
h(X)=\log |A|, \quad \mathcal{N}(X)=|A|^{2 / n} .
$$

The entropy power inequality, due to Shannon and Stam ([20], [21], cf. also [22], [23]), asserts that

$$
\mathcal{N}(X+Y) \geq \mathcal{N}(X)+\mathcal{N}(Y),
$$

for any two independent random vectors $X$ and $Y$ in $\mathbf{R}^{n}$, for which the entropy is defined. Although it is not directly equivalent to the Brunn-Minkowski inequality, it is very similar to it [23].

To judge the sharpness of the entropy power inequality, we need to keep in mind that the entropy is invariant under linear volume preserving transformation of the space, i.e., $\mathcal{N}(u(X))=\mathcal{N}(X)$ whenever $|\operatorname{det}(u)|=1$. On the other hand, the left-hand side of (7) essentially depends on "positions" of the distributions of $X$ and $Y$. Therefore, to reverse this inequality, some transformation of these random vectors is needed. Then, we have:

Theorem 3: Let $X$ and $Y$ be independent random vectors in $\mathbf{R}^{n}$ with log-concave densities. There exist linear volume preserving maps $u_{i}: \mathbf{R}^{n} \rightarrow \mathbf{R}^{n}$ such that

$$
\mathcal{N}(\tilde{X}+\widetilde{Y}) \leq C(\mathcal{N}(X)+\mathcal{N}(Y)),
$$

where $\widetilde{X}=u_{1}(X), \widetilde{Y}=u_{2}(Y)$, and where $C$ is a universal constant.

The proof of this result, as well as extensions of it and further discussion, can be found in [19].

\section{REFERENCES}

[1] J. Bourgain, "On high-dimensional maximal functions associated to convex bodies," Amer. J. Math., vol. 108, no. 6, pp. 1467-1476, 1986.

[2] K. Ball, "Logarithmically concave functions and sections of convex sets in $\mathbf{R}^{n}$," Studia Math., vol. 88, no. 1, pp. 69-84, 1988.

[3] S. Bobkov, "Isoperimetric and analytic inequalities for log-concave probability measures," Ann. Prob., vol. 27, no. 4, pp. 1903-1921, 1999.

[4] S. G. Bobkov and M. Madiman, "The entropy per coordinate of a random vector is highly constrained under convexity conditions," Preprint, 2009.

[5] T. Cover and J. Thomas, Elements of Information Theory. New York: J. Wiley, 1991.

[6] D. Hensley, "Slicing convex bodies-bounds for slice area in terms of the body's covariance," Proc. Amer. Math. Soc., vol. 79, no. 4, pp. 619625,1980

[7] V. D. Milman and A. Pajor, "Isotropic position and inertia ellipsoids and zonoids of the unit ball of a normed $n$-dimensional space," in Geometric aspects of functional analysis (1987-88), ser. Lecture Notes in Math. Berlin: Springer, 1989, vol. 1376, pp. 64-104.

[8] J. Bourgain, "On the distribution of polynomials on high-dimensional convex sets," in Geometric aspects of functional analysis (1989-90), ser. Lecture Notes in Math. Berlin: Springer, 1991, vol. 1469, pp. 127-137.
[9] G. Paouris, "On the isotropic constant of non-symmetric convex bodies," in Geometric aspects of functional analysis, ser. Lecture Notes in Math. Berlin: Springer, 2000, vol. 1745, pp. 239-243.

[10] B. Klartag, "On convex perturbations with a bounded isotropic constant," Geom. Funct. Anal., vol. 16, no. 6, pp. 1274-1290, 2006.

[11] V. D. Milman, "Inégalité de Brunn-Minkowski inverse et applications à la théorie locale des espaces normés," C. R. Acad. Sci. Paris Sér. I Math., vol. 302, no. 1, pp. 25-28, 1986.

[12] — "Isomorphic symmetrizations and geometric inequalities," in Geometric aspects of functional analysis (1986/87), ser. Lecture Notes in Math. Berlin: Springer, 1988, vol. 1317, pp. 107-131.

[13] — , "Entropy point of view on some geometric inequalities," $C . R$. Acad. Sci. Paris Sér. I Math., vol. 306, no. 14, pp. 611-615, 1988.

[14] G. Pisier, The volume of convex bodies and Banach space geometry, ser. Cambridge Tracts in Mathematics. Cambridge: Cambridge University Press, 1989, vol. 94.

[15] J. Bourgain, B. Klartag, and V. Milman, "Symmetrization and isotropic constants of convex bodies," in Geometric aspects of functional analysis, ser. Lecture Notes in Math. Berlin: Springer, 2004, vol. 1850, pp. 101115.

[16] H. Koenig and N. Tomczak-Jaegermann, "Geometric inequalities for a class of exponential measures," Proc. Amer. Math. Soc., vol. 133, no. 4, pp. 1213-1221 (electronic), 2005.

[17] B. Klartag and V. D. Milman, "Geometry of log-concave functions and measures," Geom. Dedicata, vol. 112, pp. 169-182, 2005.

[18] S. Artstein-Avidan, V. Milman, and Y. Ostrover, "The $M$-ellipsoid, symplectic capacities and volume," Comment. Math. Helv., vol. 83, no. 2 , pp. 359-369, 2008.

[19] S. G. Bobkov and M. Madiman, "Reverse Brunn-Minkowski and reverse entropy power inequalities for convex measures," Preprint, 2010.

[20] C. Shannon, "A mathematical theory of communication," Bell System Tech. J., vol. 27, pp. 379-423, 623-656, 1948.

[21] A. Stam, "Some inequalities satisfied by the quantities of information of Fisher and Shannon," Information and Control, vol. 2, pp. 101-112, 1959.

[22] M. Costa and T. Cover, "On the similarity of the entropy power inequality and the Brunn-Minkowski inequality," IEEE Trans. Inform. Theory, vol. 30, no. 6, pp. 837-839, 1984.

[23] A. Dembo, T. Cover, and J. Thomas, "Information-theoretic inequalities," IEEE Trans. Inform. Theory, vol. 37, no. 6, pp. 1501-1518, 1991 